\title{
El efecto de la participación en actividades de ocio sobre la influencia de la distancia en la elección de destinos
}

\author{
JUAN LUIS NICOLAU \\ Departamento de Marketing, UNIVERSIDAD DE ALICANTE, ESPAÑA. E-mail: \\ JL.Nicolau@ua.es
}

\begin{abstract}
RESUMEN
En la elección de destinos, la distancia presenta un efecto negativo debido al esfuerzo que supone desplazarse y a que generalmente implica mayores costes. Este artículo analiza si los individuos están dispuestos a recorrer mayores distancias para ir a un destino en el que pueden practicar, por ejemplo, actividades de ocio distintas a las que hacen el resto del año, o aumentar la frecuencia de sus actividades preferidas. La idea es que la sensibilidad a la distancia está influenciada a la hora de elegir un destino por la motivación de búsqueda de destinos que poseen determinadas actividades de ocio disponibles.
\end{abstract}

Palabras clave: Elección de destinos, sensibilidad a la distancia, actividades de ocio.

\section{When the Enrollment in Recreational Activities Changes Distance Sensitivity to Destination Choice}

\begin{abstract}
In destination choice, distance shows a negative effect because it entails an effort to get to the destination and generally implies higher costs. Accordingly, this article attempts to analyze whether people are willing to cover longer distances to go to a destination where they can change recreational patterns; e.g. do recreational activities different from those at their place of residence, or do the same leisure activities as in their home environment with higher frequency. The idea is that distance sensitivity is influenced, at the moment of choosing a destination, by the driver of searching for destinations with specific recreational activities.
\end{abstract}

Keywords: Destination Choice, Sensitivity to Distance, Leisure Activities.

Clasificación JEL: D12

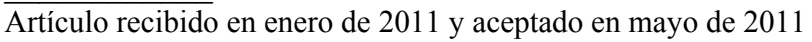

Artículo disponible en versión electrónica en la página www.revista-eea.net, ref. ə-29310 


\section{INTRODUCCIÓN}

El efecto de la distancia en la elección de destinos turísticos ha suscitado un importante interés en la literatura turística (Eryigit et al., 2010; Fredman, 2008; Lyons et al., 2009; McKercher et al., 2008; Nelson et al., 2011; Ohe, 2010; Seetanah et al., 2010). Ciertamente, como bienes de experiencia que son, los productos turísticos poseen las características de los servicios -intangibilidad, caducidad, heterogeneidad y simultaneidad-. Así, el hecho de que la producción y el consumo de los servicios se realice simultáneamente, conduce a que el cliente tiene que desplazarse al lugar donde se presta el servicio. Esta característica cobra especial relevancia cuando se trata de la elección de destinos turísticos: si bien un cliente debe desplazarse para recibir las prestaciones del servicio "peluquería", el desplazamiento inherente en los viajes turísticos para que el cliente reciba los servicios existentes en el destino no es comparable con el anterior tipo de servicio. No sólo forma parte de la experiencia turística, sino que el turista también debe invertir tiempo y esfuerzo para llegar al lugar donde se prestan los servicios. Aunque existen evidencias de que este desplazamiento no siempre ejerce un efecto disuasorio (Lise y Tol, 2002), por lo general, la literatura tiende a considerarlo como un elemento -a modo de barrera- que el turista tiene que superar para "disfrutar" del destino: es el denominado "efecto de la disminución por distancia" (distance decay effect), por el que la demanda turística decrece exponencialmente ante incrementos en la distancia (Bull, 1991).

En esta línea, cabe preguntarse qué factores pueden reducir dicho efecto disuasorio de la distancia; es decir, qué elementos hacen que un turista tenga mayor predisposición a realizar un largo trayecto. Este trabajo plantea la idea de que las actividades disponibles en el destino pueden ser un estímulo que reduzca el efecto negativo de la distancia, de modo que un individuo puede elegir uno más lejano si a cambio puede participar en determinadas actividades. En concreto, a partir de los trabajos de Carr (2002), McKercher y du Cros (2003), Brey y Lehto (2007) y McKercher (2008), el presente estudio propone que la posibilidad de incrementar -o mantener- la frecuencia de participación en actividades preferidas conduce a que los turistas reduzcan su sensibilidad a la distancia.

Con este propósito, las siguientes secciones se organizan como sigue: el apartado segundo revisa brevemente el efecto de distancia así como la participación en actividades turísticas como elemento clave en la elección de destinos; el apartado tercero presenta el diseño de la investigación, con la metodología, la muestra y los resultados. Finalmente, el cuarto apartado describe las conclusiones obtenidas. 


\section{LA DISTANCIA Y LA PARTICIPACIÓN EN ACTIVIDADES TURÍSTICAS: EFECTO EN LA ELECCIÓN DE DESTINOS}

La distancia entre el lugar de residencia habitual del individuo y los respectivos destinos es un criterio de especial relevancia debido a la marcada dimensión espacial inherente en la elección de destinos turísticos. Sin embargo, su efecto puede ser doble y en sentidos opuestos. Por una parte, la distancia -o posición geográfica relativa que ocupa el turista con respecto a los distintos destinos- es considerada una restricción o variable disuasoria de la elección del destino, ya que el desplazamiento del individuo hasta el destino supone un esfuerzo físico, temporal y monetario (Taylor y Knudson, 1976). Este resultado se obtiene en los trabajos de Wennergren y Nielsen (1970), Perdue (1986), Borgers et al., (1989), Fesenmaier (1988), Adamowicz et al. (1994), Schroeder y Louviere (1999) y Maddison (2001). Por otra parte, una perspectiva de investigación propone que la distancia puede proporcionar utilidad positiva. Así, Lise y Tol (2002) encuentran evidencias en este sentido para el mercado holandés; y en esta línea, Baxter (1980) señala que el propio viaje, como componente del producto turístico, puede proporcionar satisfacción en sí mismo, de ahí que en ocasiones se prefieran mayores distancias. De igual modo, Wolfe $(1970 ; 1972)$ evidencia que la distancia no siempre actúa como un factor disuasorio, ya que la fricción derivada de la misma desaparece cuando se sobrepasa un determinado umbral, convirtiéndose en un atributo favorable para la formación de la utilidad que proporciona un destino. Beaman $(1974 ; 1976)$ explica este comportamiento a partir de un análisis marginal de la distancia, al observar la reacción de los individuos ante cada unidad de distancia, concluyendo que cada unidad adicional recorrida ofrece menos resistencia que la anterior. Asimismo, Greer y Wall (1979) indican que un mínimo de distancia es preciso recorrer para que el turista tenga la "sensación de escapar" necesaria para obtener satisfacción en los viajes turísticos. Por tanto, el análisis de la distancia no es fácilmente predecible en la medida en que varios factores pueden influir en su efecto; en particular, y como se expondrá a continuación, en el contexto de las actividades turísticas el papel que desempeña la distancia es complejo. En este sentido, McKercher (2008) sugiere que la reducción exponencial de la demanda depende de otros factores que influyen en la selección de los destinos, tales como los deseos de participar en actividades diferentes.

En esta línea, un aspecto de interés dentro del contexto de elección de destinos es el análisis del efecto que las distintas frecuencias de participación en actividades recreativas -en el entorno habitual del individuo y en el destinopueden tener en las sensibilidades a la distancia que el turista presenta. Conocer estas relaciones es fundamental para las organizaciones que gestionan tales actividades de cara a detectar sus mejores grupos de turistas y cómo dirigirse a ellos. Ello les permitiría segmentar el mercado de acuerdo con el criterio 
"beneficio buscado por el consumidor" centrándose en aquéllos que están dispuestos a realizar mayores distancias para ir a un destino donde pueden practicar actividades distintas a aquellas realizadas en su lugar de residencia habitual, o el criterio "frecuencia de uso" dirigiéndose a personas interesadas en practicar la misma actividad recreativa con más frecuencia de lo que lo hacen en su entorno habitual. En este sentido, cabe recordar que Silberman (1985) sugiere que a medida que la distancia se incrementa, la duración de la estancia también aumenta, debido al hecho de que los costes de viaje son fijos e independientes del número de días en el destino, lo que conduce a que mayores estancias permiten a los turistas repartir dichos costes fijos sobre un período más largo. En otras palabras, un turista estará más predispuesto a realizar un trayecto más largo si está en el destino durante un mínimo de días que le compense el esfuerzo realizado en el desplazamiento. Evidentemente, si estos segmentos están más predispuestos a mayores desplazamientos porque ello les permite disfrutar de "novedad" o "incrementar la frecuencia de participación", representan grupos de consumidores atractivos para los destinos y las organizaciones ubicadas en los mismos, ya que tienden a gastar mayores cantidades debido a sus estancias más largas.

La idea es que la sensibilidad a la distancia de cada persona está influenciada, en el momento de elegir un destino, por una motivación que le empuja a buscar un destino que le permita la práctica de determinadas actividades recreativas. A su vez, estas motivaciones de participación condicionan el efecto de la cultura residual (por la que las personas muestran en el destino patrones de conducta similar a la que exhiben en su lugar de residencia habitual) y el efecto de la cultura turística (por la que dichos patrones de conducta cambian en los destinos con respecto a los de su entorno habitual) (Carr, 2002). La lógica subyacente es que las motivaciones pueden convertirse en los principales generadores de utilidad cuando se visita un destino, y de este modo, la gente estará más o menos dispuesta a realizar un esfuerzo dependiendo de sus deseos en un momento concreto. En este sentido, el efecto de la distancia sobre un destino puede cambiar en función de la motivación de la persona. Así, McKercher y du Cros (2003) encuentran que los turistas que recorren distancias cortas frente a los de distancias largas manifiestan diferentes motivaciones a la hora de elegir un destino. La teoría del comportamiento del consumidor considera que las motivaciones representa las fuerzas internas del individuo que conducen a la acción (Schiffman y Kanuk, 2007); por ello, las motivaciones son características que influyen en la elección de destinos dado que pueden actuar como factores que empujan a la realización de un viaje (Moutinho, 1987; Sirakaya, 1992; Sirakaya et al., 1996; Kim y Lee, 2002). Es importante enfatizar que la selección de un destino concreto implica el deseo de algún tipo de beneficio. Debido a esto, las motivaciones juegan un papel fundamental en la elección de destinos puesto que constituyen pensamientos internos que llevan a 
un persona a conseguir un determinado objetivo (Nahab, 1975); esto es, son las razones por las que la gente elige unas vacaciones (Santos, 1983). Estrechamente relacionadas con las motivaciones aparecen los conceptos de cultura residual y cultura turística, que Carr (2002) utiliza en su "continuo de comportamiento turístico y de ocio". La cultura residual se refiere al comportamiento que las personas muestran en su entorno habitual (los hábitos que tiene) mientras que la cultura turística influye en el comportamiento que las personas muestran en los destinos turísticos (más liberados). En medio, tienen cabida una serie de comportamientos intermedios que son influenciados y condicionados por la fuerza que cada cultura -residual o turística- ejerce (Carr, 2002). Nos basamos en este esquema para analizar los cambios en las sensibilidades a la distancia a la hora de elegir un destino en función de los distintos patrones de participación en actividades turísticas, comparando su comportamiento en los destinos y en su lugar de residencia habitual ${ }^{1}$.

El presente artículo analiza directamente las siguientes situaciones: aquellas personas que se involucran en nuevas actividades recreativas ("sin participación en el entorno habitual" pero "con participación ocasional o frecuente en el destino"), aquéllos que incrementan su frecuencia de participación ("participación ocasional en el entorno habitual" pero "participación frecuente en el destino") y aquéllos que mantienen la misma frecuencia de participación ("participación ocasional o frecuente en el entorno habitual" y "participación ocasional o frecuente en el destino"). Esta definición de comportamientos nos permite examinar si existen distintas sensibilidades a la distancia en función de los escenarios definidos por las culturas turística y residual (con diferentes frecuencias de participación) y en función del tipo de actividad recreativa.

\footnotetext{
${ }^{1}$ De acuerdo con Brey y Lehto (2007), la literatura ha dedicado escasa atención a la relación entre las actividades recreativas en el lugar de residencia del individuo y las realizadas por éste en los destinos. Estos autores explican comportamientos diferenciados a partir de los conceptos de implicación y especialización (Kyle et al., 2004; Iwasaki y Havitz, 1998, 2004; Brey y Lehto, 2007). La implicación es un estado motivacional del individuo que representa el grado de excitación e interés que una determinada situación o estímulo provoca en el sujeto (Rodríguez y Luque, 2002), y que incluso puede hacer que el consumidor encuentre diversión en el propio proceso de compra (Mittal y Lee, 1989). En este sentido, dicha excitación, interés y diversión puede hacer percibir y sentir la distancia a recorrer menos gravosa. Por su parte, en el concepto de especialización, una persona pasa por varias etapas en una escala de "interés por una actividad", empezando como un participante genérico y llegando al nivel de especialista. A partir de estos conceptos, Brey y Lehto (2007) encuentran que cuanto más implicado está una persona en una actividad recreativa diaria, más tiende a participar en la misma actividad cuando se va de vacaciones.
} 


\section{DISEÑO DE LA INVESTIGACIÓN}

\subsection{Metodología}

La metodología propuesta que permite estimar y explicar las sensibilidades individuales a la distancia a partir de decisiones reales, consta de dos etapas: i) estimación de las sensibilidades individuales a la distancia a través de Modelos Logit con Coeficientes Aleatorios; y ii) aplicación de un análisis de regresión.

\subsubsection{Estimación de las sensibilidades individuales a la distancia}

Para estimar los parámetros individuales -es decir, las sensibilidades individuales a la distancia- de un Modelo Logit con Coeficientes Aleatorios, se aplican métodos de estimación Bayesiana ${ }^{2}$ en un contexto de elección de destinos. Se utilizan estos modelos debido a su capacidad para tratar la heterogeneidad no observada de los turistas, y a que es un modelo flexible que permite representar distintos patrones de correlación entre las distintas alternativas. Siguiendo el enfoque formal de Train (2009), la función de utilidad se define como $U_{i n}=\beta_{n} d_{i s t_{i}}+\varepsilon_{\text {in }}$ donde dist $_{i}$ es la distancia desde el origen del individuo al destino $i ; \beta_{n}$ es el parámetro de la distancia para el individuo $n$ que representa la sensibilidad personal; es decir, permite identificar la sensibilidad individual al atributo "distancia"; y $\varepsilon_{i n}$ es un término aleatorio que se distribuye iid valor extremo. La probabilidad de elegir $i$ por el individuo $n$ condicionado al parámetro $\beta$ se expresa como

$$
P\left(i / \text { dist }_{i}, \beta\right)=\frac{\exp \left\{\beta_{n} \text { dist }_{i}\right\}}{\sum_{j=1}^{J} \exp \left\{\beta_{n} \text { dist }_{j}\right\}}
$$

Dado que $\beta_{n}$ no es conocida, la probabilidad de elección de una alternativa es la integral de la expresión anterior sobre la distribución de $\beta$ :

$$
P\left(i / \text { dist }_{i}, \theta\right)=\int P\left(i / \text { dist }_{i}, \beta\right) g(\beta / \theta) d \beta
$$

donde $g(\beta / \theta)$ es la distribución del vector de parámetros aleatorios $\beta$ en la población de individuos, y $\theta$ son los parámetros de esta distribución (media y varianza). Con la aplicación de procedimientos Bayesianos obtenemos la sensibilidad a la distancia $\beta_{n}$ del individuo $n$.

2 Train (2001) señala las ventajas de utilizar procedimientos bayesianos sobre los procedimientos clásicos: i) se evitan los problemas habituales de máximos globales y locales, ya que no se basan en la maximización de ninguna función de probabilidad; y ii) se obtienen estimaciones consistentes y eficientes bajo condiciones más flexibles. 


\subsubsection{Identificación de la relación entre los patrones de actividades recreativas} y la sensibilidad a la distancia

Para analizar la relación entre los distintos patrones de actividades recreativas y la sensibilidad individual a la distancia, se aplica el análisis de regresión clásico. Sea $x_{n s}$ un grupo de variables $s$ que describen el comportamiento recreativo del individuo $n$ (en términos de frecuencia de participación) que se proponen para explicar la sensibilidad a la distancia $\beta_{n}$, y $\delta_{s}$ los coeficientes que reflejan los efectos de estas variables en dicha sensibilidad. Así, se obtiene la regression:

$$
\beta_{n}=\sum_{s=1}^{S} \delta_{n} x_{n s}+u_{t}
$$

donde la perturbación aleatoria $u_{n}$ sigue una distribución normal de media cero y varianza $\sigma_{u}$.

\subsection{Muestra, datos y variables}

Para alcanzar nuestro objetivo, utilizamos información sobre comportamiento de elección turístico obtenido de una encuesta de carácter nacional desarrollada por el Centro de Investigaciones Sociológicas (CIS). Ello obedece a las siguientes razones: i) La disponibilidad de información referida al comportamiento turístico de elección tanto en los destinos como en el entorno habitual; y ii) La encuesta va dirigida a una muestra de individuos (mayores de 18 años), obtenida en origen, que evita el sesgo de selección característico de las muestras recogidas en los destinos, que conduce a un análisis más preciso de la demanda turística. La muestra se extrae de una población de individuos mayores de edad, siguiendo un muestreo polietápico, estratificado por conglomerados, con selección de las unidades primarias -municipios- y de las unidades secundarias secciones censales- de forma proporcional. Se realizan entrevistas personales en los domicilios con cuestionario estructurado. De la muestra inicial de 3.781 individuos, seleccionamos los 2.127 que salen de vacaciones, de modo que se puede comparar las frecuencias de participación en los destinos con las del entorno habitual.

De cara a hacer el modelo operativo, se definen las variables utilizadas y se identifican la variable dependiente y las independientes.

1. Variable dependiente. Para representar el destino seleccionado por un individuo, se utilizan 50 variables dummy para las provincias españolas. En el modelo de regresión la variable dependiente es la sensibilidad a la distancia estimada en el modelo de elección.

2. Variable independiente para el modelo de elección: Distancia al destino. Para este estudio, se utiliza la distancia real en kilómetros en línea con 
Wennergren y Nielsen (1970), Stopher y Ergün (1979), Moutinho y Trimble (1981), Peterson et al. (1983), Perdue (1986), Borgers et al. (1989), Fesenmaier (1988), Adamowicz et al. (1994), Dellaert et al. (1997) y Schroeder \& Louviere (1999). Su empleo implica la construcción de una matriz de orígenes y destinos de orden 50x50, en la que se muestran los kilómetros entre cada origen y cada destino. Esta información sobre distancias se obtiene de la Guía Campsa Interactiva (tomando las capitales de provincia como puntos de referencias) ${ }^{3}$.

3. Variable independiente para el modelo de regresión: Variables de frecuencia. Los encuestados proporcionan información sobre su frecuencia de participación en actividades recreativas durante el período vacacional en los destinos y durante el resto del año en su entorno habitual. Se mide mediante una escala ordinal de tres niveles (actividad practicada frecuentemente, ocasionalmente y no practicada) en línea con Brey y Letho (2007). Con esta información construimos cinco variables relevantes para este estudio: i) Novedad frecuente, que representa una actividad en la que la persona no participa a lo largo del año pero que práctica con frecuencia durante el período vacacional; ii) Novedad ocasional, cuando la actividad no es practicada en el entorno habitual pero sí practicada ocasionalmente en el destino; iii) Frecuencia aumentada, cuando se trata de una actividad en la que el individuo participa ocasionalmente durante todo el año y frecuentemente durante las vacaciones; iv) Actividad frecuente todo el año, cuando se practica frecuentemente todo el año y durante las vacaciones; y v) Actividad ocasional todo el año, referida a una actividad practicada ocasionalmente en el entorno habitual y en el destino.

En concreto, las actividades recreativas analizadas son las siguientes: a) visita a museos, catedrales y otros monumentos; b) visita de lugares de singular

\footnotetext{
${ }^{3}$ Con la finalidad de introducir variabilidad en el atractivo de los diferentes destinos, también utilizamos el precio como variable de control, el cual a su vez es uno de los factores más determinantes en la elección de destinos. Para su medición, autores como Eymann y Ronning (1992) y Usach (1999) consideran que el método correcto de reflejar los precios de un mercado turístico para un contexto de elección consiste en la comparación de los precios de los destinos con los del mercado de origen y con los de los destinos competidores. En este sentido, Eymann y Ronning (1992) utilizan los diferenciales de las paridades de compra entre los orígenes y los destinos, obtenidos de los correspondientes índices de precio al consumo. Asimismo, Morley (1994) demuestra que el índice de precios al consumo de una región geográfica es un buen indicador de los precios turísticos, mostrando una alta correlación entre los dos. En línea con estos autores, nuestro estudio mide los precios de los destinos "provincias" a través de los diferenciales de los índices de precio al consumo entre los orígenes y los destinos, publicados por el Instituto Nacional de Estadística, que representa el coste de vida de cada origen y cada destino. El uso de esta variable conlleva la elaboración de una matriz de orden 50x50 que cruza los orígenes con los destinos.
} 
belleza natural; c) vídeo y fotografía; d) visita de áreas naturales protegidas (parques naturales); e) observación de la flora y fauna; y f) actividades tradicionales locales (artesanía, actividades agrícolas, etc.).

\section{RESULTADOS}

Como primer paso, utilizamos los procedimientos Bayesianos para estimar los coeficientes (sensibilidades) de la variable "distancia" para cada individuo, mediante Modelos Logit de Coeficientes Aleatorios. La Tabla 1 muestra los resultados globales que representan las preferencias de un individuo medio.

\section{Tabla 1}

Influencia de la distancia en la elección de destinos

(Errores estándar entre paréntesis)

\begin{tabular}{|c|c|c|}
\hline & Distancia & Variable de control: Precio \\
\hline $\boldsymbol{b}$ & $-0.398^{\mathrm{a}}$ & $-0.222^{\mathrm{a}}$ \\
& $(0.014)$ & $(0.021)$ \\
\hline \multirow{2}{*}{ SD $(\beta)$} & $\begin{array}{c}0.146^{\mathrm{a}} \\
(0.012)\end{array}$ & $\begin{array}{c}0.056^{\mathrm{a}} \\
(0.012)\end{array}$ \\
\hline
\end{tabular}

$\mathrm{a}=$ prob $<0.1 \%$

Fuente: Elaboración propia.

En relación con el impacto de la distancia, se observa que es significativa a un nivel de 0,001 con un signo negativo. Ello lleva a caracterizarla como un factor disuasorio en la elección de destinos (resultado análogo se obtiene para la variable de control "precio"). Sin embargo, cabe remarcar el hecho que el parámetro de la varianza del coeficiente $(\operatorname{SD}(\beta))$ es significativo, lo que implica que la distancia tiene un efecto diferenciado entre los individuos de la muestra y, por tanto, una determinada distancia no supone la misma reducción en la utilidad para todos los individuos. En consecuencia, el efecto diferenciado detectado para la distancia sugiere que existe una gran diversidad de sensibilidades en el mercado en línea con el análisis a nivel de segmentos realizados por McKercher (2008). Las Gráficas 1-6 muestran esta dispersión para cada actividad, obteniéndose incluso valores positivos para algunos individuos de acuerdo con la argumentación de Baxter (1980) y los resultados de Lise y Tol (2002). Ello implica que esta heterogeneidad detectada puede ser potencialmente explicada por las variables de frecuencia de participación en actividades. De hecho, la Figura 1 presenta una primera exploración gráfica de modo que para cada actividad, los patrones de frecuencia de los turistas tienen valores de sensibilidad a la distancia distintos. 


\section{Gráfica 1}

Histograma de la sensibilidad a la distancia en la actividad "visita a museos, catedrales y otros monumentos"

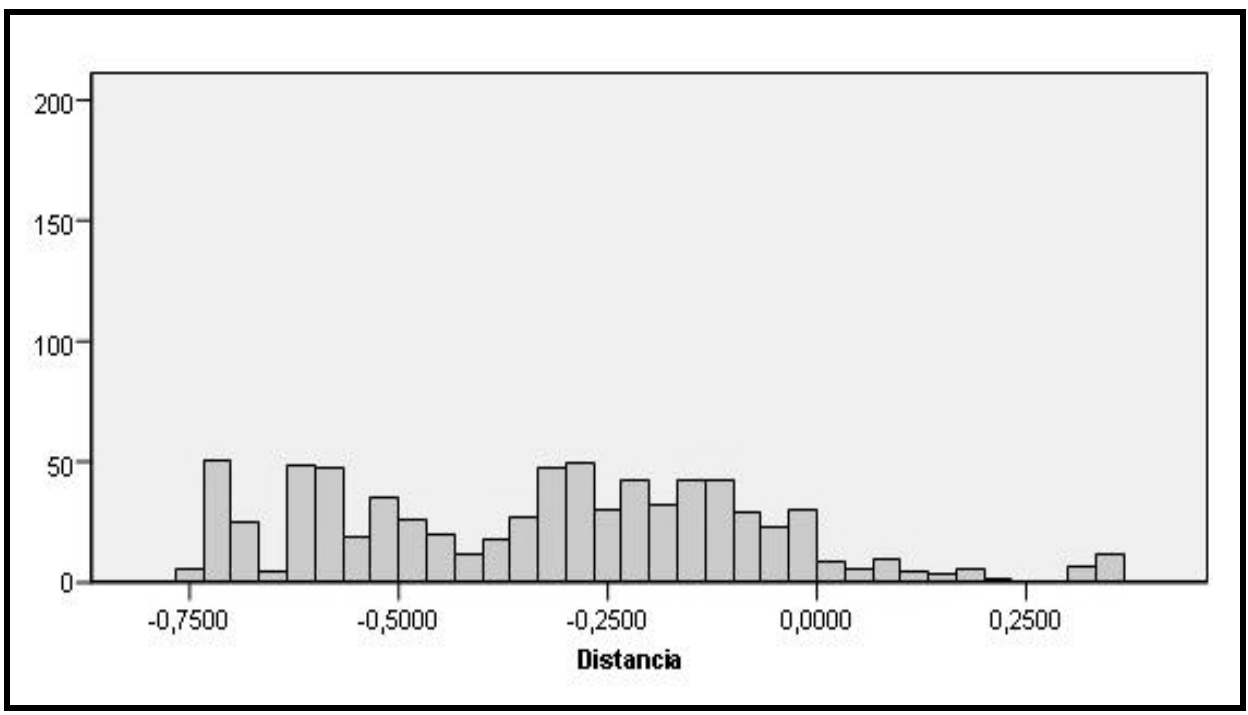

Fuente: Elaboración propia.

\section{Gráfica 2}

Histograma de la sensibilidad a la distancia en la actividad "visita de lugares de singular belleza natural"

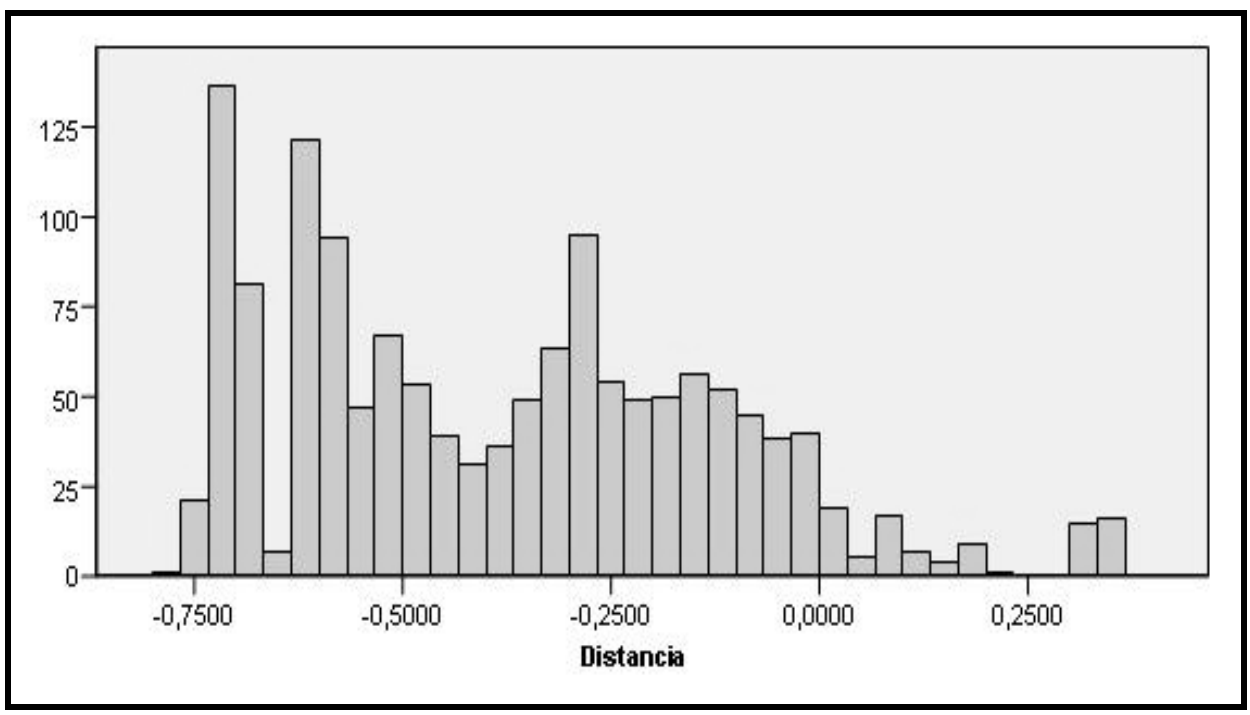

Fuente: Elaboración propia. 


\section{Gráfica 3}

Histograma de la sensibilidad a la distancia en la actividad "vídeo y fotografía"

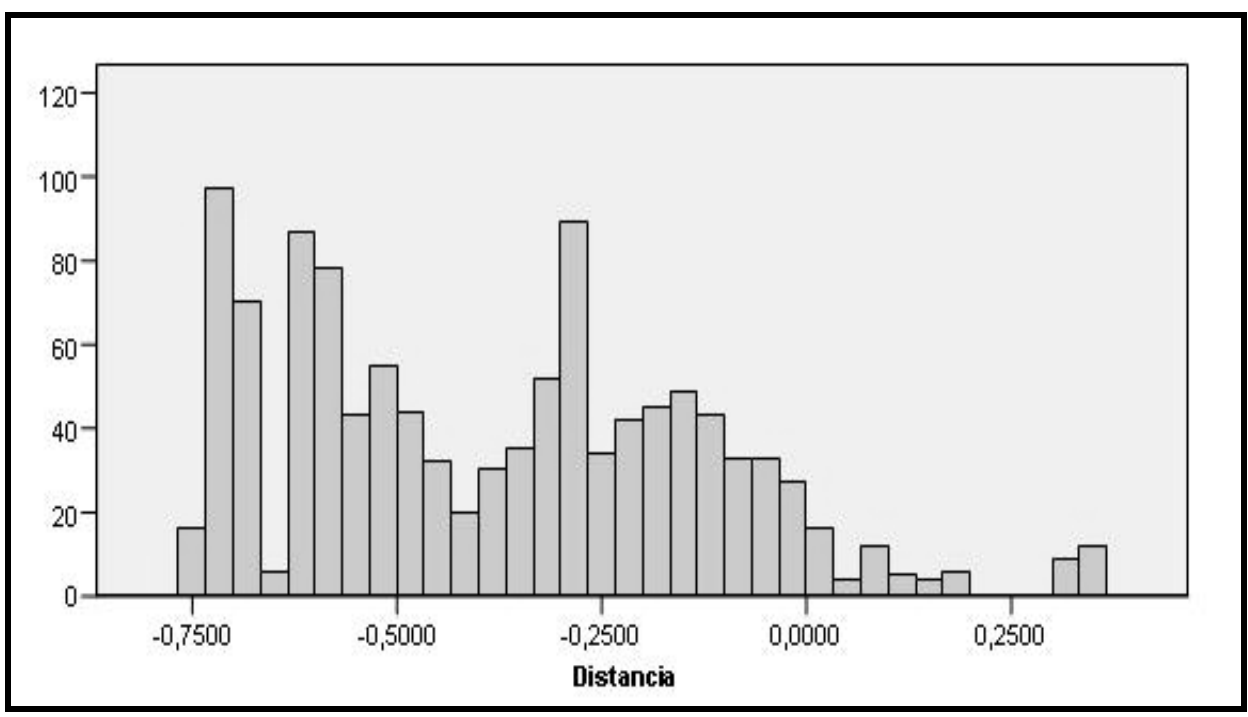

Fuente: Elaboración propia.

\section{Gráfica 4}

Histograma de la sensibilidad a la distancia en la actividad "visita de áreas naturales protegidas"

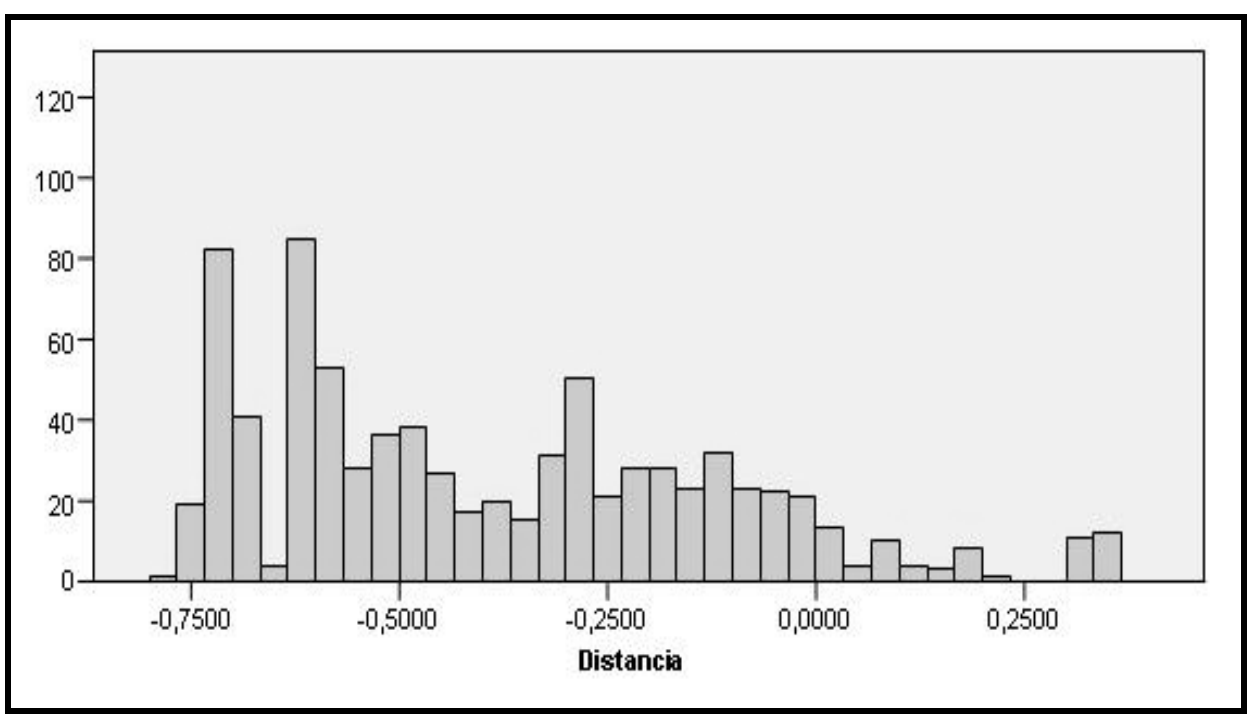

Fuente: Elaboración propia. 


\section{Gráfica 5}

Histograma de la sensibilidad a la distancia en la actividad "observación de la flora y fauna"

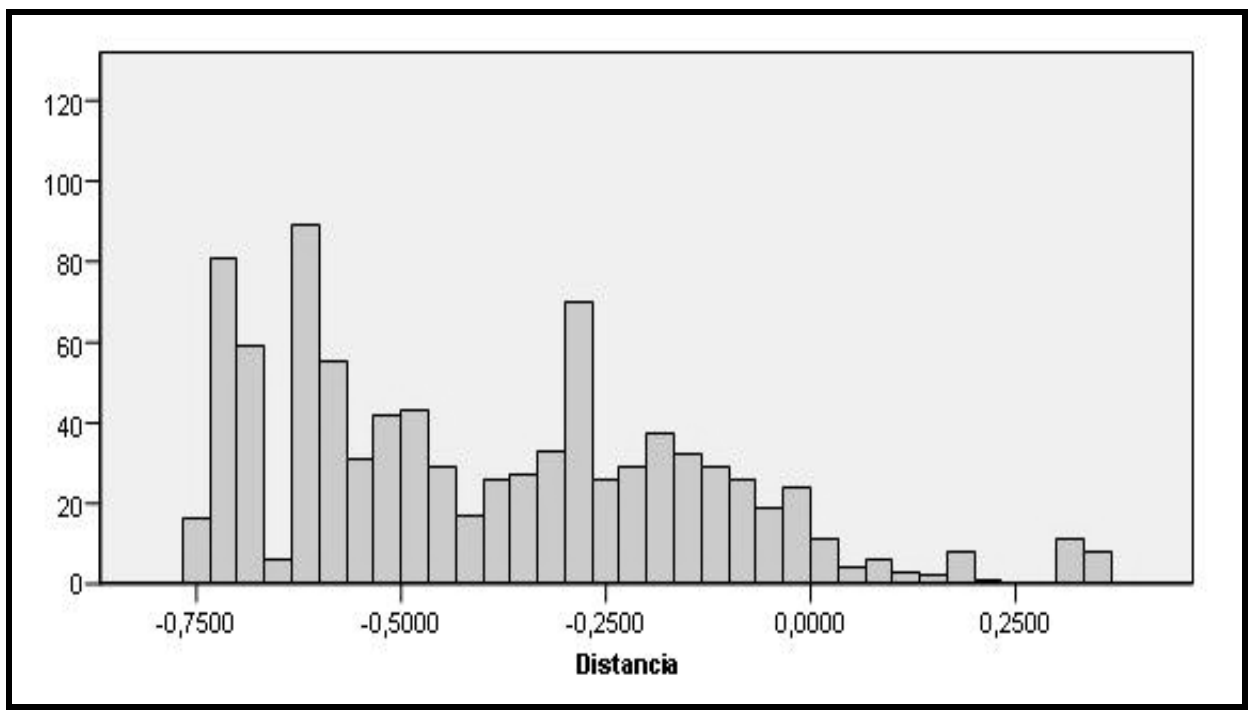

Fuente: Elaboración propia.

\section{Gráfica 6}

Histograma de la sensibilidad a la distancia en "actividades tradicionales locales"

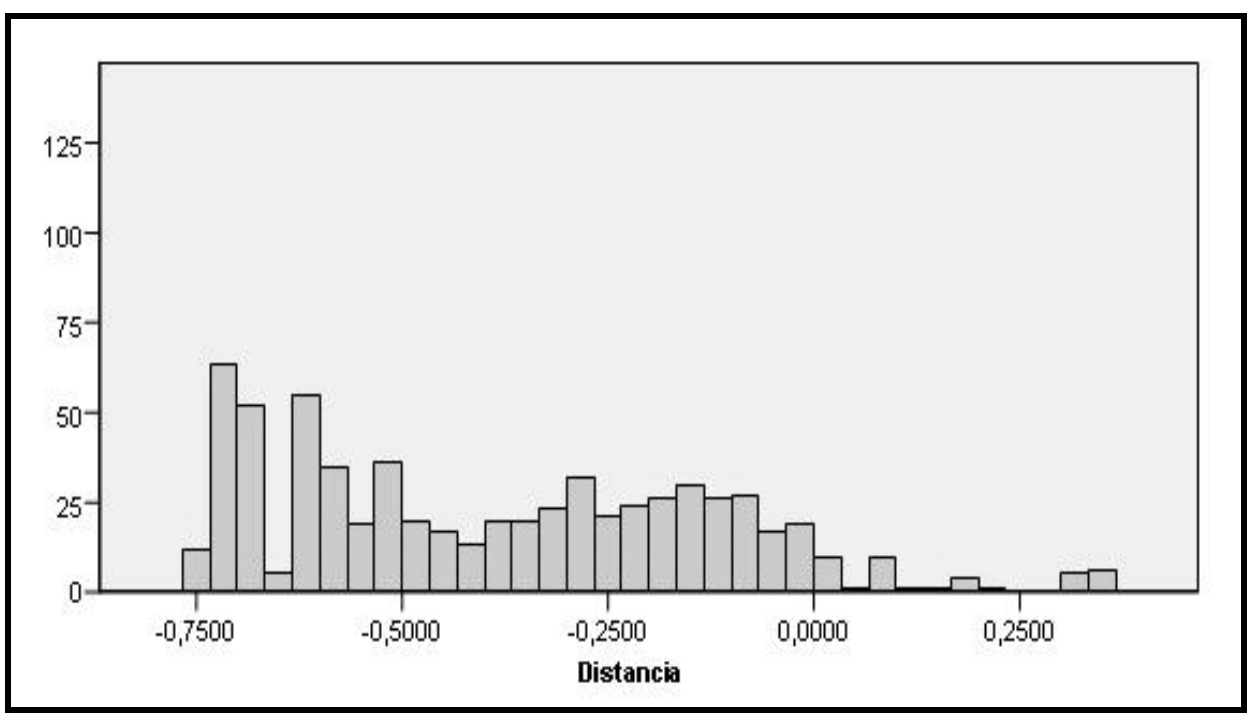

Fuente: Elaboración propia. 
Figura 1

Gráficas de sensibilidad a la distancia por tipos de actividad y patrones de frecuencia

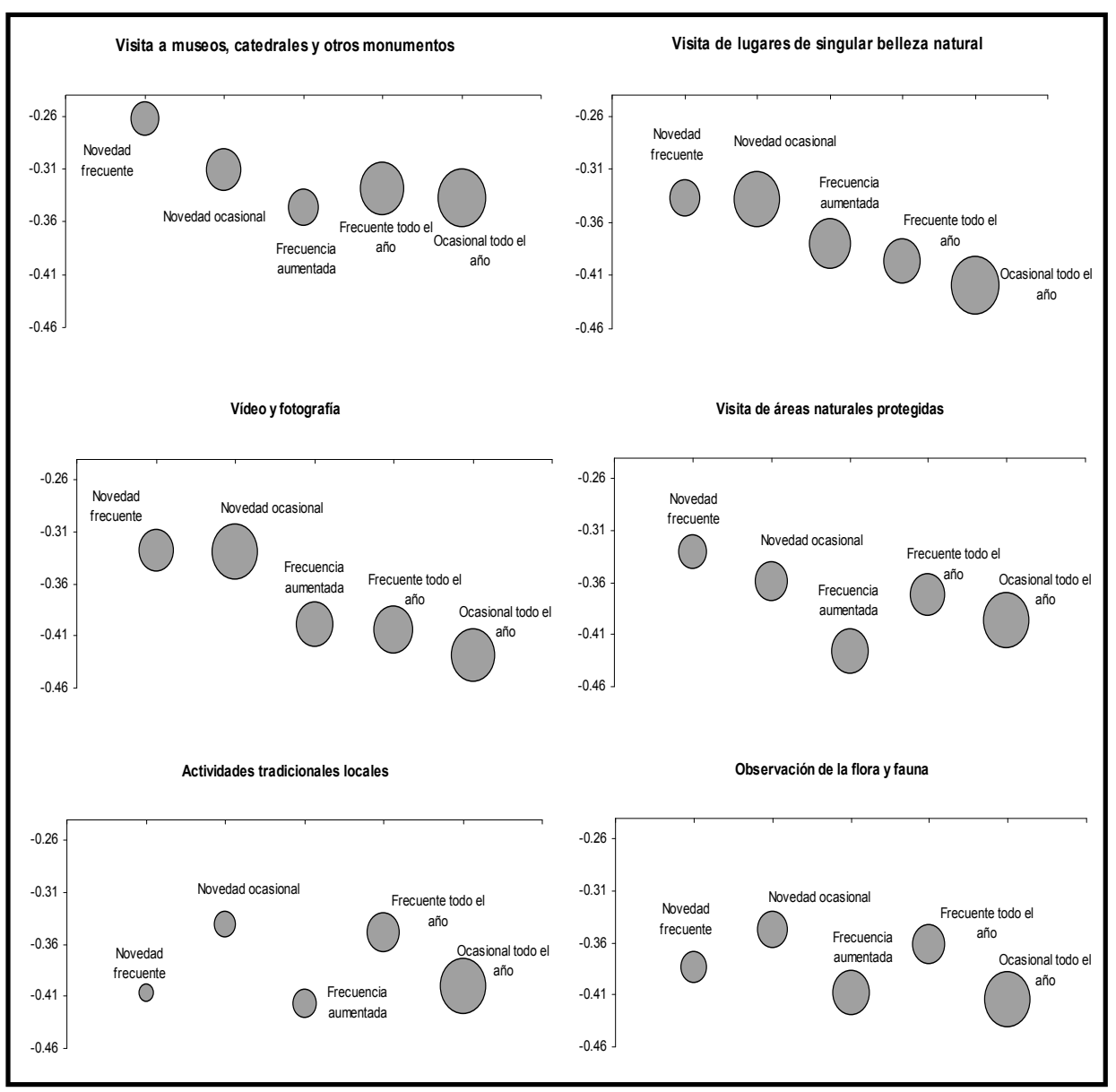

Fuente: Elaboración propia.

A continuación, estas sensibilidades individuales a la distancia se utilizan en el análisis de regresión como variable dependiente para examinar de qué manera las variables de frecuencia propuestas pueden influir en las mismas. Los resultados obtenidos son los siguientes (Tabla 2): La actividad de visitar museos, catedrales y otros monumentos presenta un signo significativo y positivo para todas las variables de frecuencia. En concreto, los parámetros de novedad frecuente y ocasional muestran que la "cultura turística" hace que los individuos estén más predispuestos a realizar distancias más largas si pueden disfrutar de esta actividad durante sus vacaciones. Obsérvese que aquellos que siguen el patrón de novedad frecuente tienen un parámetro mayor, lo que significa que 
son menos sensibles a la distancia que aquellos con el patrón novedad ocasional. Motivados por la "cultura residual", aquellos que siguen el patrón frecuente y ocasional todo el año, tienen predisposición hacia los destinos de larga distancia siempre y cuando puedan visitar museos, catedrales y otros monumentos. Finalmente, el parámetro de la frecuencia aumentada presenta el segundo mayor impacto en la reducción de la sensibilidad a la distancia. Existe, por tanto, una mezcla de efectos -cultura turística y residual-: por una parte, un individuo intenta seguir los hábitos de los que obtiene satisfacción (cultural residual) y, por otra parte, esta tendencia se intensifica por la liberalización del individuo durante sus vacaciones (sin preocupaciones, más tiempo libre, etc.), lo que les permite disfrutar de esta actividad deseada con mayor frecuencia (cultura turística).

Tabla 2

Efecto de la frecuencia de participación en la sensibilidad a la distancia

(Errores estándar entre paréntesis)

\begin{tabular}{|c|c|c|c|c|c|c|c|}
\hline & $\begin{array}{l}\text { Novedad } \\
\text { frecuente }\end{array}$ & $\begin{array}{l}\text { Novedad } \\
\text { ocasional }\end{array}$ & $\begin{array}{l}\text { Frecuencia } \\
\text { aumentada }\end{array}$ & $\begin{array}{c}\begin{array}{c}\text { Frecuente } \\
\text { todo el } \\
\text { año }\end{array} \\
\end{array}$ & $\begin{array}{c}\text { Ocasional } \\
\text { todo el } \\
\text { año }\end{array}$ & Constante & $\begin{array}{c}\text { Estadístico } \\
F\end{array}$ \\
\hline $\begin{array}{l}\text { Visita a museos, } \\
\text { catedrales y otros } \\
\text { monumentos }\end{array}$ & $\begin{array}{l}0.169^{a} \\
(0.029)\end{array}$ & $\begin{array}{l}0.102^{a} \\
(0.020)\end{array}$ & $\begin{array}{l}0.128^{a} \\
(0.024)\end{array}$ & $\begin{array}{l}0.102^{a} \\
(0.026)\end{array}$ & $\begin{array}{l}0.109^{a} \\
(0.018)\end{array}$ & $\begin{array}{l}-0.442^{a} \\
(0.007)\end{array}$ & $19.377^{a}$ \\
\hline $\begin{array}{l}\text { Visita de lugares } \\
\text { de singular } \\
\text { belleza natural }\end{array}$ & $\begin{array}{l}0.114^{a} \\
(0.023)\end{array}$ & $\begin{array}{l}0.057^{c} \\
(0.020)\end{array}$ & $\begin{array}{l}0.107^{a} \\
(0.018)\end{array}$ & $\begin{array}{l}0.072^{a} \\
(0.019)\end{array}$ & $\begin{array}{l}0.030^{d} \\
(0.017)\end{array}$ & $\begin{array}{l}-0.449^{a} \\
(0.011)\end{array}$ & $10.095^{a}$ \\
\hline Fotografía y vídeo & $\begin{array}{l}0.094^{\mathrm{a}} \\
(0.023)\end{array}$ & $\begin{array}{c}0.019 \\
(0.020)\end{array}$ & $\begin{array}{l}0.101^{\mathrm{a}} \\
(0.017)\end{array}$ & $\begin{array}{l}0.033^{d} \\
(0.020)\end{array}$ & $\begin{array}{l}-0.000 \\
(0.019)\end{array}$ & $\begin{array}{l}-0.426^{a} \\
(0.009)\end{array}$ & $9.071^{a}$ \\
\hline $\begin{array}{l}\text { Visita de áreas } \\
\text { naturales } \\
\text { protegidas }\end{array}$ & $\begin{array}{c}0.060 \\
(0.028)\end{array}$ & $\begin{array}{l}0.054^{d} \\
(0.022)\end{array}$ & $\begin{array}{l}0.069^{b} \\
(0.024)\end{array}$ & $\begin{array}{l}-0.012 \\
(0.022)\end{array}$ & $\begin{array}{c}0.018 \\
(0.018)\end{array}$ & $\begin{array}{l}-0.411^{a} \\
(0.007)\end{array}$ & $3.368^{b}$ \\
\hline $\begin{array}{l}\text { Observación de la } \\
\text { flora y fauna }\end{array}$ & $\begin{array}{c}0.022 \\
(0.029) \\
\end{array}$ & $\begin{array}{l}0.053^{d} \\
(0.023) \\
\end{array}$ & $\begin{array}{c}0.066^{\mathrm{b}} \\
(0.024) \\
\end{array}$ & $\begin{array}{c}0.002 \\
(0.020) \\
\end{array}$ & $\begin{array}{l}-0.003 \\
(0.017) \\
\end{array}$ & $\begin{array}{l}-0.407^{a} \\
(0.008) \\
\end{array}$ & $2.507^{\mathrm{c}}$ \\
\hline $\begin{array}{l}\text { Actividades } \\
\text { tradicionales } \\
\text { locales }\end{array}$ & $\begin{array}{l}-0.023 \\
(0.043)\end{array}$ & $\begin{array}{l}0.058^{\mathrm{C}} \\
(0.023)\end{array}$ & $\begin{array}{l}0.062^{d} \\
(0.032)\end{array}$ & $\begin{array}{l}-0.007 \\
(0.030)\end{array}$ & $\begin{array}{c}0.002 \\
(0.017)\end{array}$ & $\begin{array}{l}-0.405 \\
(0.007)\end{array}$ & $2.054^{d}$ \\
\hline
\end{tabular}

$a=$ prob $<0.1 \% ; b=$ prob $<1 \% ; c=$ prob $<5 \% ; d=$ prob $<10 \%$

Fuente: Elaboración propia.

Para la actividad de visita de lugares de singular belleza natural se obtienen resultados similares: todas las variables de frecuencia tienen parámetros significativos y positivos. En particular, los parámetros de novedad frecuente y ocasional indican, al igual que antes, que la "cultura turística" conduce a los turistas a emprender distancias mayores para experimentar esta actividad durante sus vacaciones. Asimismo, aquellos que siguen el patrón novedad frecuente muestran un parámetro positivo mayor (son menos sensibles a la distancia que aquellos con el patrón novedad ocasional). El mismo comportamiento se detec- 
ta para el patrón frecuente y ocasional todo el año, aunque en este caso motivados por la "cultura residual", teniendo el parámetro de frecuente todo el año más peso que el ocasional todo el año en términos de insensibilidad a la distancia. El parámetro de frecuencia aumentada también tiene el segundo mayor impacto en la reducción de la sensibilidad a la distancia (la mezcla de efectos de cultura turística y residual se aplica igualmente en este caso).

Para las actividades relacionadas con el vídeo y la fotografía, se obtienen parámetros significativos y positivos para novedad frecuente, frecuencia aumentada y frecuencia todo el año. Concretamente, bajo la novedad frecuente, la gente tiene un incentivo a visitar destinos lejanos. El resultado es coherente con los obtenidos para la actividad de "visitar lugares de singular belleza natural" en el que los turistas están dispuestos a viajar más lejos para visitar áreas con vistas especiales y realizar fotografías de tales paisajes. Aquellos turistas que buscan aumentar la frecuencia (de manera ocasional en su lugar de residencia a frecuente en el destino) y para aquellos que desean mantener altas frecuencias en ambos lugares, existe una clara reducción en la sensibilidad a la distancia. Estos turistas presentan una propensión a realizar largar distancias si ello les permite practicar la actividad a la que son aficionados. Obsérvese que, de acuerdo con los resultados obtenidos en las tres variables significativas y positivas (novedad frecuente, frecuencia aumentada y frecuente todo el año), esta actividad -vídeo y fotografía- está claramente influenciada por ambas culturas, turística y residual.

Para las actividades "visita de áreas naturales protegidas", "observación de flora y fauna" y "actividades locales tradicionales", se detectan parámetros significativos y positivos para los patrones novedad ocasional y frecuencia aumentada. En relación con la novedad ocasional, el resultado obtenido significa que estas actividades -no realizadas en su lugar de residencia durante el año- suponen un incentivo para ir a un destino que está lejano. Respecto a la frecuencia aumentada, los que se involucran en estas actividades durante el año tienden a especializarse en ellas, en línea con Brey y Lehto (2007), de tal forma que los turistas son más selectivos a la hora de elegir dónde realizarlas. Este rasgo selectivo implica que las personas pueden estar más dispuestas a mayores desplazamientos, reduciéndose así el efecto negativo de la distancia, siendo más insensibles a la misma.

\section{CONCLUSIONES}

Un aspecto interesante en el marco de la elección de destinos es el examen del efecto que las distintas frecuencias de participación en actividades de ocio en el entorno habitual y en el destino- tienen en la sensibilidad a la distancia. La idea en la que se basa este artículo es que la sensibilidad individual a la distancia está influenciada por las motivaciones que lleva a las personas a 
buscar destinos que les permiten participar en actividades recreativas específicas; y lo que es más, estas motivaciones tienen un efecto en la influencia que la cultura residual y la cultura turística ejercen en el comportamiento del consumidor. En este sentido, el objetivo del artículo consiste en analizar si la sensibilidad individual a la distancia está afectada por las distintas frecuencias de participación en actividades recreativas en el entorno habitual del individuo y en el destino.

Con este propósito, medimos e identificamos las sensibilidad a la distancia individuo a individuo- a partir de elecciones reales (la sensibilidad individual a la distancia se estima para cada persona observando el destino que realmente elige). La formalización operativa empleada para estimar dichas sensibilidades sigue el Modelo Logit con Coeficientes Aleatorios, y para detectar el efecto de la motivación propuesta, se aplica un análisis de regresión. La aplicación empírica llega a la conclusión general de que la cultura residual y la turística influyen en la sensibilidad a la distancia en las actividades "visitar museos, catedrales y otros monumentos", "visitar lugares de singular belleza natural", "actividades de vídeo y fotografía", "visitar áreas naturales protegidas", "observación de flora y fauna" y "actividades locales tradicionales".

Nótese que para la misma actividad, los dos efectos pueden tener un impacto en la sensibilidad a la distancia, pero con distintas magnitudes. Por ejemplo, la sensibilidad a la distancia de los turistas que participan en "visitar museos, catedrales y otros monumentos" se reduce en mayor medida para una persona que no participa en esta actividad durante todo el año y tratan de aprovecharla al máximo en sus vacaciones (buscadores de novedad frecuente), que para una persona que participa en la actividad durante todo el año. Asimismo, dentro del mismo efecto -cultura turística o residual-, las influencias de las variables de frecuencia pueden cambiar. Así, para el efecto de la cultura turística en la actividad "visitar lugares de singular belleza natural", los buscadores de "novedad frecuente" tienen una reducción mayor en la sensibilidad a la distancia que aquellos que tienen el patrón "novedad ocasional" (en concreto, el parámetro del primero es el doble del segundo).

En cuanto a las implicaciones de gestión, se pueden mencionar las siguientes: dado que la existencia de diversidad de sensibilidades a la distancia se ha confirmado, una implicación importante es que, el conocimiento de la estructura de preferencias de las personas -individuo a individuo- en términos de su respuesta a la distancia permite que los gestores encuentren el destino apropiado para cada persona, así como la formación de segmentos con similares "preferencias hacia la distancia". Por ejemplo, podrían segmentar el mercado centrándose en el beneficio buscado a través de la identificación de aquellas personas que están dispuestas a recorrer mayores distancias para ir a un destino donde pueden practicar actividades recreativas diferentes a las de su entorno 
habitual, o en la frecuencia de uso, buscando personas que quieren incrementar durante sus vacaciones, la frecuencia de participación de las actividades que ya hacen en su entorno habitual. Evidentemente, dado que estos turistas están dispuestos a mayores desplazamientos para disfrutar de la novedad o la frecuencia aumentada, representan segmentos de mercado interesantes en la medida que los viajeros de largas distancias tienden a gastar más debido a sus estancias más largas.

El análisis se ha basado en las preferencias de las personas, y evidentemente las preferencias son elementos claves en la elección de destinos. Además, la estimación de los parámetros individuales de la función de utilidad de cada individuo revela su estructura de preferencias lo que permite al analista operar con información precisa sobre cada persona. En un momento en el que la gente cada vez más exige servicios adaptados a sus requerimientos específicos, el conocimiento del perfil de cada individuo permite a las organizaciones configurar la oferta de productos más adecuados. La identificación de personas con más o menos sensibilidad a la distancia de los destinos, con un énfasis específico en su capacidad de proporcionarle su actividad deseada, es crucial para los gestores de los destinos. En esta línea, estos gestores pueden:

1. conocer su clientela en términos de preferencias. El mercado turístico está caracterizado por una marcada heterogeneidad entre los turistas, lo que implica la existencia de una gran diversidad de necesidades y deseos; y no sólo entre turistas sino también "dentro" de cada turista, ya que sus preferencias pueden cambiar en función de la temporada (Cuccia, 2009). Este hecho obliga a las organizaciones turísticas a considerar la segmentación como un elemento clave para la estrategia de Marketing (López et al., 2002). Es más, en este contexto -en el que la demanda es cada vez más exigente-, el turista busca una prestación de servicios adaptada a sus necesidades específicas, y ser tratado como un cliente único. Este hecho explica que en los últimos años se haya abierto paso, tanto a nivel académico como empresarial, el Marketing de relaciones y la aplicación del CRM (Customer Relationship Management), que tienen como principio fundamental el tratamiento personalizado -uno a uno-, de los turistas. Ciertamente, el turista actual "bien informado" ya no se conforma con los "destinos de siempre", y busca lugares nuevos y poco frecuentes (OMT, 2001). Además, la procedencia de los mismos es cada vez más variada: recuérdese que al continuo crecimiento de los flujos turísticos proyectado por la OMT (2001) -con independencia de las fluctuaciones características del sector (Otero et al., 1999)-, se debe sumar el nuevo impacto a nivel internacional del flujo turístico emitido por los países del BRIC. Ello conduce a una clara necesidad de obtener un conocimiento de los "distintos" turistas con sus "diversos" requerimientos. Conocer el comportamiento de los turistas para mantener una relación 
continua y a largo plazo implica observar sus decisiones de compra, es decir, las elecciones que realiza. En este sentido, Bronner y De Hoog (1985) señalan que el modo en que los individuos toman sus decisiones es un aspecto relevante para ser utilizado como base para la segmentación de mercados. En este contexto, este trabajo ha identificado los procesos de decisión individuo a individuo, turista a turista, a partir de la estimación de la función de utilidad individual de cada uno de ellos. La función de utilidad de un turista permite explicar por qué un individuo elige un determinado destino (y no otro) o averiguar qué factores son determinantes para que una persona elija un tipo de viaje específico. Todo ello, supone el punto de partida para la inmediata aplicación del CRM tanto a nivel de destinos como de empresas turísticas.

2. desarrollar productos adecuados con los atributos correctos. De acuerdo con el informe Amadeus (2009), el 80\% de los ingresos de las agencias de viajes procede del 60\% de sus productos (lejos de la típica regla 80-20). Este dato sugiere que la especialización es una necesidad; especialización que está fundamentada en nichos de mercados. Es decir, un turista tradicional de sol y playa representa, hoy en día, un perfil muy genérico de turista: hoy nos podemos encontrar con un turista de sol y playa que puede estar dispuesto a ir a una playa más lejana si allí puede practicar su actividad favorita, por lo que el producto turístico no sería "sol y playa" sino "sol y playa más actividad favorita".

3. diseñar campañas promocionales dirigidas al segmento escogido haciendo hincapié en las características apropiadas. Una vez identificado el/los mejor/es segmento/s para un destino o empresa turística, y una vez que se han adaptado sus productos turísticos a los segmentos, queda la tarea de comunicarles la existencia y excelencias de la oferta. De hecho, para definir con precisión las campañas promocionales del destino turístico, desde el punto de vista público y privado, es imprescindible conocer los perfiles de turistas (Dávila et al., 2002). Dada la segregación de las preferencias de la demanda en la que ya no sólo sirve llegar al nivel de segmentos sino que hay que bajar al nivel de subsegmentos o nichos de mercados, en principio, no parecería sencillo llegar de manera individualizada a los miembros de dichos subsegmentos. No obstante, en este punto hay que recordar que de acuerdo con el informe de la Comisión del Mercado de Telecomunicaciones (CMT, 2010), casi el 50\% de las ventas a través de Internet se refieren al mundo de los viajes, destacando que la industria turística lidera el comercio electrónico. En este sentido, el tratamiento individualizado de la comunicación se convierte en algo posible y factible: no sólo las nuevas tecnologías permiten, técnicamente, adaptar la comunicación de las organizaciones a cada cliente, sino que existe una 
clara predisposición por parte de los turistas a hacer uso de estas nuevas tecnologías.

Para concluir, el análisis presentado en este artículo permite a las organizaciones que gestionan actividades turísticas la identificación de los segmentos adecuados, perfilar dichas actividades e implementar sus estrategias de promoción. En esta línea, a la luz de estos resultados, la medida clave a adoptar por parte de estas organizaciones y los propios destinos debería basarse en la implementación del CRM: no sólo porque los turistas piden un tratamiento individualizado sino porque en la actualidad es relativamente factible desarrollarlo. Ello permitiría segmentar los mercados a nivel de microsegmentos -individuo a individuo-, y por tanto, adaptar su oferta en cualquier dimensión de interés, como los precios, el canal de distribución, el tipo de hotel demandado, el modo de comunicarse con el cliente, o como en el caso de la aplicación empírica de este trabajo, en función de la dimensión espacial "distancia" y la existencia de determinadas "actividades". Así, dado que la frecuencia de participación -en el entorno habitual y en el destino- influye en la sensibilidad a la distancia, la disponibilidad de ciertas actividades recreativas ayuda a reducir el efecto negativo de la distancia, ya que los turistas motivados por la oportunidad de probar una actividad no practicada durante el año, de mantener su frecuencia de participación o de incrementarla, pueden convertirse en menos sensibles a la distancia. Es más, este efecto puede condicionar variables relevantes para los destinos tales como duración de la estancia y los gastos totales.

\section{REFERENCIAS BIBLIOGRÁFICAS}

ADAMOWICZ, W., LOUVIERE, J. Y WILLIAMS, M. (1994). "Combining Revea-led and Stated Preference Methods for Valuing Environmental Amenities", Journal of Environmental Economics and Management, 26, 271-292.

AMADEUS (2009). El viajero experto. Madrid: Amadeus.

BAXTER, M.J. (1980). "The Interpretation of the Distance and Attractiveness Components in Models of Recreational Trips", Geographical Analysis, 11(3), 311-315.

BEAMAN, J. (1974). "Distance and the 'Reaction' to Distance as a Function of Distance", Journal of Leisure Research, 6, summer, 220-231.

BEAMAN, J. (1976). "Corrections Regarding the Impedance of Distance Functions for Several $g(d)$ Functions", Journal of Leisure Research, 49-52.

BORGERS, A.W. J., VAN DER HEIJDEN, R.E.C.M. Y TIMMERMANS, H.J.P. (1989). "A Variety Seeking Model of Spatial Choice-behaviour", Environment and Planning A, 21, 1037-1048. 
BREY, E.T. Y LEHTO, X.Y. (2007). "The relationship between daily and vacation activities", Annals of Tourism Research, 34(1), 160-180.

BRONNER, F. Y HOOG, R. (1985). "A Recipe for Mixing Decision Ingredients", European Research, 13, 109-115.

BULL, A. (1991). The economics of travel and tourism. Melbourne: Pitman.

CARR, N. (2002). "The tourism-leisure behavioural continuum", Annals of Tourism Research, 29(4), 972-986.

CMT, COMISIÓN DEL MERCADO DE TELECOMUNICACIONES (2010). "Los ingresos del comercio online en España superan pro primera vez los 1.500 millones", Nota de prensa, 22 de febrero de 2010. Madrid: Comisión del Mercado de Telecomunicaciones.

CUCCIA, T. (2009). A Contingent Ranking Study on the Preferences of Tourists across Seasons, Estudios de Economía Aplicada, 27(1), 159-174.

DÁVILA, D.; RODRÍGUEZ, S. Y RODRIGUEZ, A. (2002). "La promoción turística espacial dentro de un destino vacacional homogéneo. Una aplicación a Canarias" Estudios de Economía Aplicada, 20(1), 45-60.

DELLAERT, B.G.C., BORGERS, A.W.J. Y TIMMERMANS, H. J.P. (1997). "Conjoint Models of Tourist Portfolio Choice: Theory and Illustration", Leisure Sciences, 19, 31-58.

ERYIGIT, M., KOTIL, E. Y ERYIGIT, R. (2010). "Factors affecting international tourism flows to Turkey: A gravity model approach", Tourism Economics 16(3), 585-595.

EYMANN, A. Y RONNING, G. (1992). "Discrete Choice Analysis of Foreign Travel Demand" in Vosgerau, H.J., ed., European Integration in the World Economy. Studies in International Economics and Institutions, Berlin: Springer.

EYMANN, A. Y RONNING, G. (1997). "Microeconometric Models of Tourists' Destination Choice", Regional Science and Urban Economics, 27, 735-761.

FESENMAIER, D.R. (1988). "Integrating Activity Patterns into Destination Choice Models", Journal of Leisure Research, 20(3), 175-191.

FREDMAN, P. (2008). "Determinants of visitor expenditures in mountain tourism", Tourism Economics, 14(2), 297-311.

GREER, T., Y WALL, G. (1979). "Recreational hinterlands: A theoretical and empirical analysis", en G. Wall (Ed.), Recreational land use in Southern Ontario, Department of Geography Publication Series \#14 (pp. 227-246). Waterloo, Canada: Waterloo University.

IWASAKI, Y. Y HAVITZ, M. (1998). "A path analystic model of the relationships between involvement, psychological commitment, and loyalty", Journal of Leisure Research, 30, 256-280.

IWASAKI, Y. Y HAVITZ, M. (2004). "Examining relationshipcs between leisure involvement, psychological commitment and loyalty to a recreation agency", Journal of Leisure Research, 36, 45-72. 
KIM, S. Y LEE, CH. (2002). "Push and Pull Relationships", Annals of Tourism Research, 29(1), 257-260.

KYLE, G., GRAEFE, A., MANNING, R. Y BACON, J. (2004). "Predictors of behavioural loyalty among hikers along the Appalachian Trail”, Leisure Science, 29, 99-118.

LISE, W., Y TOL, R. S. J. (2002). "Impact of climate on tourist demand", Climatic Change, 55(4), 429-449.

LÓPEZ, P.; TRUJILLO, F. Y ALARCÓN, P. (2002). Imagen turística de las Comunidades Autónomas españolas ante una demanda segmentada. Estudios de economía aplicada, 20(3), 627-649.

LYONS, S., MAYOR, K. Y TOL, R.S.J. (2009). "Holiday destinations: Understanding the travel choices of Irish tourists", Tourism Management, 30(5), 683-692.

MADDISON, D. (2001). "In search of warmer climates? The impact of climate change on flows of British tourists", Climatic Change, 49(1), 193-208.

MCKERCHER, B. (2008). "Segment transformation in urban tourism", Tourism Management, 29(6), 1215-1225.

MCKERCHER, B., CHAN, A. Y LAM, C. (2008). "The impact of distance on international tourist movements", Journal of Travel Research, 47(2), 208-224.

MCKERCHER, B., Y DU CROS, H. (2003). Testing a cultural tourism typology. International Journal of Tourism Research, 5(1), 45-58.

MITTAL, B. Y LEE, M.S. (1989). "A causal model of consumer involvement”, Journal of Economic Psychology, 10, 363-389.

MORLEY, C.L. (1994). "The Use of CPI for Tourism Prices in Demand Modelling", Tourism Management, 15(5), 342-346.

MOUTINHO, L. (1987). "Consumer Behaviour in Tourism”, European Journal of Marketing, 21(10), 1-44.

MOUTINHO, L. Y TRIMBLE, J. (1991). “A Probability of Revisitation Model: The Case of Winter Visits to the Grand Canyon", The Service Industries Journal, 11(4), 439-457.

NAHAB, S. (1975). Tourism Management, London: Tourism International Press, Londres.

NELSON, L.A., DICKEY, D.A. Y SMITH, J.M. (2011). "Estimating time series and cross section tourism demand models: Mainland United States to Hawaii data", Tourism Management, 32(1), 28-38.

OHE, Y. (2010). "Evaluating integrated on-farm tourism activity after rural road inauguration - The case of pick-your-own fruit farming in Gunma, Japan", Tourism Economics, 16(3), 731-753.

OMT (2001) Tourism 2020 Vision. Madrid: World Tourism Organization.

OTERO, J.M Y EQUIPO SAETA (1999). "Las fluctuaciones cíclicas del turismo en Andalucía", Estudios de Economía Aplicada, 13, 129-142.

PERDUE, R.R. (1986). "Traders and Nontraders in Recreational Destination Choice", Journal of Leisure Research, 18(1), 12-25. 
PETERSON, G. L., DWYER, J.F. Y DARRAGH, A. J. (1983). "A Behavioral Urban Recreation Site Choice Model”, Leisure Sciences, 6(1), 61-81.

RODRÍGUEZ, M.A. Y LUQUE, T. (2002). "El papel moderador de la implicación hacia el mensaje en la eficacia publicitaria de los anuncios con y sin famosos", XIV Encuentros de Profesores Universitarios de Marketing, Granada, 259-276.

SANTOS, J.L. (1983). "La Decisión de Compra del Turista-Consumidor", Estudios Turísticos, 79, 39-53.

SCHIFFMAN, L.G. Y KANUK, L.L (2007). Consumer Behavior, Prentice Hall, Englewood Cliffs, New Jersey.

SCHROEDER, H.W. Y LOUVIERE, J. (1999). "Stated Choice Models for Predicting the Impact of User Fees at Public Recreation Sites", Journal of Leisure Research, 31(3), 300-324.

SEETANAH, B., DURBARRY, R. Y RAGODOO, J.F.N. (2010). "Using the panel cointegration approach to analyse the determinants of tourism demand in South Africa", Tourism Economics, 16(3), 715-729.

SILBERMAN, J. (1985). "A Demand Function for Lenght of Stay: The Evidence from Virginia Beach", Journal of Travel Research, 23(4), 16-23.

SIRAKAYA, E. (1992). Modeling Vacation Destination Choice Decisions: Development of an Instrument, Clemson University Masters Thesis, South Carolina: Clemson University.

SIRAKAYA, E., MCLELLAN, R.W. Y UYSAL, M. (1996). "Modeling Vacation Destinations Decisions: A Behavioural Approach", Journal fo Travel \& Tourism Marketing, 5(1/2), 57-75.

STOPHER, P.R. Y ERGÜN, G. (1979). "Population Segmentation in Urban Recreation Choices", Transportation Research, 59-65.

TAYLOR, CH. E. Y KNUDSON, D.M. (1976). "Area Preferences of Midwestern Campers", Journal of Leisure Research, 5, 39-48.

TRAIN, K.E. (1998). "Recreation Demand Models with Taste Differences over People", Land Economics, 74, 2, 230-239.

TRAIN, K.E. (2001). "A Comparison of Hierarchical Bayes and Maximum Simulated Likelehood for Mixed Logit”, Documento de Trabajo, Universidad de California, Berkeley.

TRAIN, K.E. (2009). Discrete Choice Methods with Simulation, Nueva York: Cambridge University Press.

USACH, J. (1999). "Un Modelo de Demanda Turística Interna para la Economía Española", Papers de Turisme, 25, 59-100.

WENNERGREN, E.B. Y NIELSEN, D.B. (1970). "Probability Estimates of Recreation Demands", Journal of Leisure Research, 2, 112-122.

WOLFE, R.I. (1970). Communication, Journal of Leisure Research, 2(1),85-87.

WOLFE, R.I. (1972). "The Inertia Model”, Journal of Leisure Research, 4, 73-76 